\title{
Isolation and Identification of Multi-Drug Resistant Acinetobacter baumannii from a tertiary health care centre of Bangladesh
}

\author{
Kartik Dhar ${ }^{1}$, Tareque Mahmud ${ }^{1}$, Rocky Chowdhury ${ }^{1 *}$, Sanzida Kulsum², \\ Tumpa Dhar ${ }^{3}$

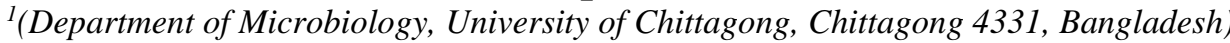 \\ ${ }^{2}$ (Department of Soil Science, University of Chittagong, Chittagong 4331, Bangladesh) \\ ${ }^{3}$ (Comilla Medical College Hospital, Comilla, Bangladesh)
}

\begin{abstract}
Acinetobacter baumannii has emerged over the last decade as a significant opportunistic pathogen and primarily associated with hospital-acquired infections. Increasing multidrug resistance pattern of A. baumannii makes it among the most difficult antimicrobial-resistant Gram-negative bacilli to control and treat. The aim of this study was to isolate and identify A. baumannii from clinical samples and to determine their antimicrobial resistance pattern to commonly prescribed drugs to find out Multi-Drug Resistant A. baumannii $(M D R A B)$. Nine different clinical samples were collected from patients admitted to Comilla Medical College Hospital (CoMCH). A. baumannii were isolated and identified based on their growth, physiological, and biochemical characteristics. Their antibiograms were studied through standard disk diffusion method, and antibiotic susceptibility patterns were interpreted. Meropenem, Cephalexin, Ampicillin, Gentamycin, Tetracycline, and Chloramphenicol were used to evaluate the sensitivity of the isolates. Three A. baumannii isolates were recovered from different clinical samples. Though the isolates showed similar growth and physiological characteristics along with similar biochemical profiles, they differ considerably in their sensitivity against several antibiotics. With an exception to tetracycline, A. baumannii W2 found to exhibit remarkable resistance against all the test antibiotics including meropenem. On the other hand, both A. baumannii $C 2$ and A. baumannii C3 showed similar resistance pattern. Both were also MDRAB showing resistance to cephalexin, ampicillin, and tetracycline. The recovery of MDRAB including meropenem-resistant A. baumannii from different clinical specimens, and their antibiotic resistance pattern hint emergence of a formidable pathogen of nosocomial origin. The findings of the study urge revision and up-gradation of current patient maintenance practices in health care providing centres of our country to limit the prevalence of antibiotic resistant $A$. baumannii.
\end{abstract}

Keywords: Acinetobacter baumannii, Multi-Drug Resistant, Nosocomial Infection

\section{Introduction}

Acinetobacter spp. is a group of Gram-negative, non-fermentative, non-motile, and oxidase-negative bacilli. The most clinically significant species of the Acinetobacter genus is A. baumannii causing over $90 \%$ of infections. Infections due to this bacterium are colligated with significant mortality [1]. A. baumannii is a commensal bacteria in healthcare facilities and has become one of the most significant microorganisms causing nosocomial infections and hospital outbreaks in last few decades [2]. It is an important infectious agent that is responsible for nosocomial infections among immune-suppressed patients accounting 5\% of Gram-negative infections [3]. Its environmental resiliency and abroad range of resistance factors' combinations facilitate to be a successful nosocomial pathogen [4]. A worldwide statistical study on A. baumannii remarked significant rise in antimicrobial resistance between 2004 and 2009 [5]. Antimicrobial resistant A. baumannii has become a global problem [6]. Some clinicians believe that the recovery of A. baumannii from the hospitalized patient bespeaks serious sickness, with an associated mortality of around 30\% [7]. A. baumannii strains resisting minimum three antibiotic classes are considered as multidrug-resistant A. baumannii (MDRAB) [8]. The main mechanisms for confabbing resistance to different antibiotic classes in A. baumannii include multidrug efflux pumps, $\beta$-lactamases, permeability defects, aminoglycoside-modifying enzymes, and target sites' alteration [9, 10]. Variety and coexistence of resistance mechanisms in this bacterium consequence in multiple drug resistance and can create troubles in treatment [9, 11-13]. Immunosuppressed patients, patients with severe inherent diseases, and those gone through invasive processes and subjected to broad-spectrum antibiotics are vulnerable to MDRAB infections [14]. Completely working antibiotic choices available to treat hospitalacquired infections with MDRAB are critically limited [15]. The rapid distribute of MDRAB in hospitals has been authenticated in various reports $[16,17]$.

Also in Bangladesh, A. baumannii is a major concern among the doctors, researchers and practitioners. Different researches have been conducted about $A$. baumannii infections and its resistance to different antimicrobials. Recovery of MDRAB isolates from different clinical samples has been reported in several 
reports in Bangladesh [18-22]. In a study conducted at the Dhaka Medical College Hospital (DMCH) reported by Khatun [21], 25 A. baumannii isolates were recovered from endotracheal aspirate samples of patients admitted to the ICU of DMCH. $96 \%$ of them (24 out of 25) showed multi-drug resistance. In another analysis carried in Square Hospitals Ltd. (SHL) reported by Mannan [22], 210 specimens were collected from the patients with lower respiratory tract infections hospitalized in the ICU of SHL. They reported A. baumannii as the most dominant pathogen among recovered isolates and $90 \%$ of the A. baumannii strains were MDRAB.

The goal of this investigation was isolation and identification of MDRAB from different types of patients with prolonged hospitalization account in different units of CoMCH. Three A. baumannii isolates were recovered from wound swab, blood, urine, and sputum samples; and their resistance pattern to some prescribed antimicrobials had also been ascertained.

\subsection{Sampling}

\section{Materials And Methods}

Clinical Samples were collected from CoMCH during August 2016. All the nine samples (3 wound swab, 3 sputum, 2 blood, and 1 urine samples)were collected from patients of different units with long ( 3 months) history of hospitalization. Swabs from wounds were collected after removing gauge bandage roll for the dressing of wounds. Then the swab sticks' tips were placed into the tubes containing sterile buffer peptone water. Blood, urine, and sputum samples were also obtained in the sterile buffer peptone water tubes according to their respective collection procedures, aseptically capped, placed in the cooler box, and immediately transferred to the laboratory [23, 24].

\subsection{Isolation and identification of $A$. baumannii}

One loopful culture from each tube of the enriched buffer peptone water was streaked on MacConkey agar surface. Plates were incubated at $37^{\circ} \mathrm{C}$ for 24 hours. White color non-lactose fermenter colonies from incubated plates were isolated and purified. Suspected isolates were subjected to catalase and oxidase test as presumptive tests. Catalase positive and oxidase negative presumptive A. baumannii were then subjected to physiological and biochemical profiling to confirm their identity.

\subsection{Antibiotic susceptibility test}

The disk diffusion technique of Kirby-Bauer [25] was employed to assess antibiotic susceptibility patterns of the selected isolates. We selected commercially available standardized antibiotic discs of Meropenem $(10 \mu \mathrm{g})$, Cephalexin $(30 \mu \mathrm{g})$, Ampicillin $(10 \mu \mathrm{g})$, Gentamycin $(10 \mu \mathrm{g})$, Tetracycline $(30 \mu \mathrm{g})$, and Chloramphenicol $(30 \mu \mathrm{g})$. The bacterial suspensions were prepared with nutrient broth from subcultures, and the McFarland standard 0.5 was used for adjusting the turbidity of the suspensions. A cotton swab was dipped into the culture preparation and streaked across the Muller-Hinton agar surface to get uniform inocula. Discs of the test antibiotics were then set on the seeded plates' surface using sterile forceps through the appropriate procedure and kept for 30 minutes at $4^{\circ} \mathrm{C}$ to diffuse antibiotic on the media surface. Then the plates were incubated for 24 hours at $37^{\circ} \mathrm{C}$. The diameter of the clear zones of inhibition were measured after incubation. Values were recorded, and susceptibility status was interpreted from the standard tables of the Clinical and Laboratory Standards Institute (CLSI) [26].

\section{Result And Discussion}

A. baumannii is an emerging pathogen causing legion worldwide outbreaks [27]. A. baumannii is spotted among the most problematic nosocomial pathogenic micro-organisms [6, 28]. Several studies reported recognizing different health care facilities in Bangladesh as breeding grounds of pathogenic A. baumannii [21, 22]. Several authors of Bangladesh cited about the recovery of MDRAB strains from clinical samples from Bangladesh [18-22].

In this study, the presence of MDRAB from few patients admitted to different units of $\mathrm{CoMCH}$ was investigated. The isolation of bacterial strains from the samples and streaking them on the MacConkey agar surfaces was the first step on isolation. Ten white color non-lactose fermenter colonies were suspected as $A$. baumannii. However, several enteric bacteria like Salmonella spp., Proteus spp., Yersinia spp., Shigella spp., and Pseudomonas aeruginosa produce similar colonies on MacConkey agar [29]. For differentiation between the target organism and enteric pathogens with similar growth characteristics on MacConkey, ten suspected isolates (4 from wound swabs, 4 from sputum, 1 from blood, and 1 from urine) were subjected to presumptive catalase and oxidase test. Only three (designated as W2, C2, and C3) of them were presumed as A. baumanni based on their hallmark catalase-positive and oxidase-negative reactions. Decisive morphological, cultural and biochemical behaviour of the three isolateswere investigated for identification purpose that involved comparing 
Isolation and Identification of Multi-Drug Resistant Acinetobacter baumannii from a tertiary health care centre of Bangladesh

and contrasting the recorded characteristics (Table 1) with the standard description provided in Bergey's Manual of Determinative Bacteriology, $8^{\text {th }}$ edition [30].

Table 1: Physiological and Biochemical behaviour of A. baumannii isolates

\begin{tabular}{|c|c|c|c|c|c|}
\hline \multirow[t]{2}{*}{ Category } & \multirow{2}{*}{\multicolumn{2}{|c|}{ Test }} & \multicolumn{3}{|c|}{ Behavior of the isolates } \\
\hline & & & A. baumannii $\mathrm{W} 2$ & A. baumannii $\mathrm{C} 2$ & A. baumannii $\mathrm{C} 3$ \\
\hline \multirow{18}{*}{$\begin{array}{l}\text { Biochemical } \\
\text { characteristics }\end{array}$} & \multicolumn{2}{|l|}{ Indole } & - & - & - \\
\hline & \multicolumn{2}{|l|}{ Citrate } & + & + & + \\
\hline & \multicolumn{2}{|l|}{$\mathrm{H}_{2} \mathrm{~S}$ production } & - & - & - \\
\hline & \multicolumn{2}{|l|}{ Nitrate reduction } & - & - & - \\
\hline & \multicolumn{2}{|l|}{ Oxidase } & - & - & - \\
\hline & \multicolumn{2}{|l|}{ Urease } & - & - & + \\
\hline & \multicolumn{2}{|l|}{ Catalase } & + & + & + \\
\hline & \multicolumn{2}{|l|}{ Starch hydrolysis } & - & - & - \\
\hline & \multicolumn{2}{|l|}{ Gelatin hydrolysis } & - & - & - \\
\hline & \multicolumn{2}{|l|}{ Esculin hydrolysis } & - & - & - \\
\hline & \multirow[t]{8}{*}{ Acid from: } & Arabinose & - & - & - \\
\hline & & Sorbitol & - & - & - \\
\hline & & Xylose & + & + & + \\
\hline & & Mannitol & - & - & - \\
\hline & & Fructose & - & - & - \\
\hline & & Sucrose & - & - & - \\
\hline & & Mannose & - & - & - \\
\hline & & Maltose & - & + & - \\
\hline $\begin{array}{l}\text { Physiological } \\
\text { characteristics }\end{array}$ & \multicolumn{2}{|l|}{ Motility } & - & - & - \\
\hline \multirow[t]{2}{*}{$\begin{array}{l}\text { Colony } \\
\text { characteristics }\end{array}$} & \multicolumn{2}{|l|}{$\begin{array}{l}\text { White colony on } \\
\text { MacConkey Agar }\end{array}$} & + & + & + \\
\hline & \multicolumn{2}{|l|}{ Fluorescent pigmentation } & - & - & - \\
\hline
\end{tabular}

"+" indicates positive result; "_" indicates negative result

The presence of $A$. baumannii in clinical samples indicates the suboptimal condition of healthcare facilities as it is an opportunistic pathogen [31]. Moreover, it is a grievous sign as detrimental effects have been found in nearly every study evaluating the contribution of $A$. baumannii to final consequences of patients [9]. Most MDRAB outbreaks have been attributed to environmental origins; hence, recovery of A. baumannii from our samples suggest the hospital lacks standard operating facilities that allows the growth of the serious pathogen. Significant effort should be devoted, without any delay, to the identification of reservoir, the introduction of rigorous hygienic practices, proper isolation of the infected individuals, prevention of cross contamination and regular surveillance of the pathogens' presence and drug resistance patterns.

We studied the susceptibility of the target isolates against some significant antibiotics. Among three $A$. baumannii isolates, A. baumannii W2 exhibited resistance to all the representative antibiotics including Meropenem, except Tetracycline (Figure 1a and Table 2).

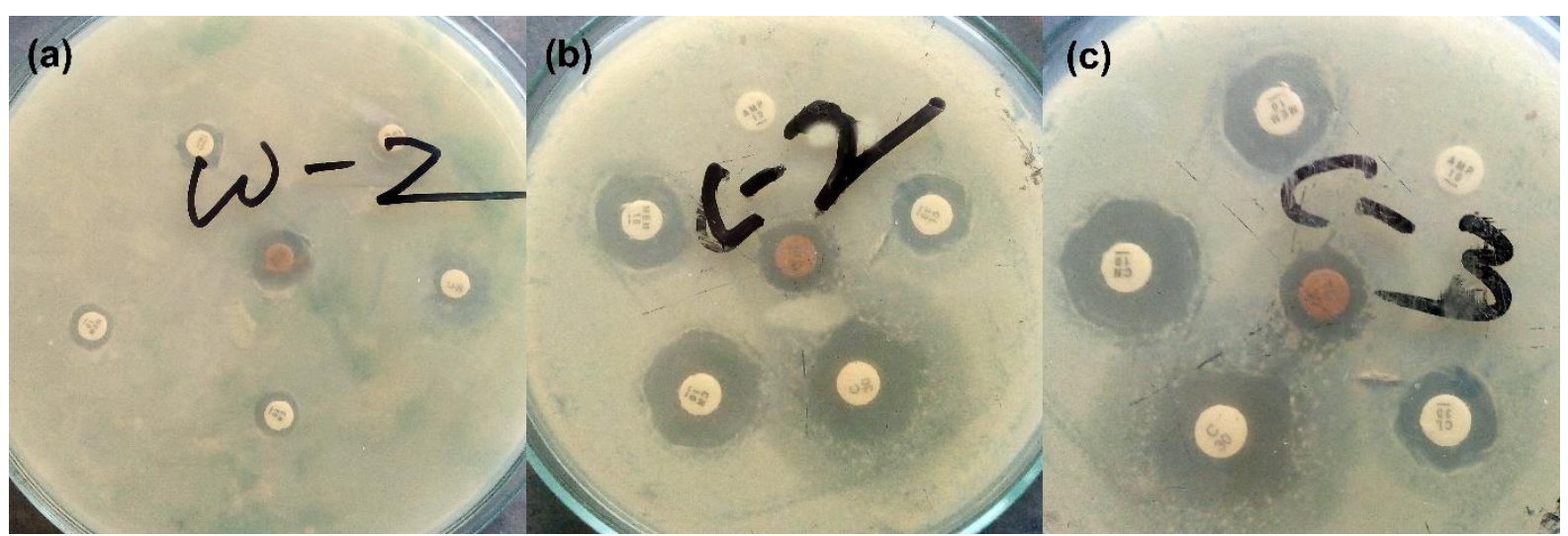

Fig. 1: Antibiotic susceptibility patterns of A. baumannii W2 (a), A. baumannii C2 (b), and A. baumannii C3 (c) against Meropenem $(10 \mu \mathrm{g})$, Cephalexin $(30 \mu \mathrm{g})$, Ampicillin $(10 \mu \mathrm{g})$, Gentamycin $(10 \mu \mathrm{g})$, Tetracycline $(30$ $\mu \mathrm{g})$, and Chloramphenicol $(30 \mu \mathrm{g})$ 
Isolation and Identification of Multi-Drug Resistant Acinetobacter baumannii from a tertiary health care centre of Bangladesh

Table 2: Antibiotic sensitivity patterns of the A. baumannii isolates against test antibiotics

\begin{tabular}{|c|c|c|c|c|c|c|c|c|c|c|}
\hline \multirow[t]{3}{*}{ Antibiotic } & \multirow{3}{*}{$\begin{array}{l}\text { Antibiotic } \\
\text { Code }\end{array}$} & \multicolumn{9}{|c|}{ Susceptibility } \\
\hline & & \multicolumn{2}{|c|}{ A. baumannii W2 } & \multicolumn{2}{|c|}{ A. baumannii C2 } & \multicolumn{2}{|c|}{ A. baumannii C3 } & \multicolumn{3}{|c|}{$\begin{array}{l}\text { Reference Value } \\
\text { (mm) }\end{array}$} \\
\hline & & Pattern & $\begin{array}{c}\text { Zone of } \\
\text { Inhibition } \\
\text { (mm) }\end{array}$ & Pattern & $\begin{array}{c}\text { Zone of } \\
\text { Inhibition } \\
\text { (mm) }\end{array}$ & Pattern & $\begin{array}{c}\text { Zone of } \\
\text { Inhibition } \\
\text { (mm) }\end{array}$ & $R^{1}$ & $I^{2}$ & $S^{3}$ \\
\hline Meropenem $(10 \mu \mathrm{g})$ & MEM & $\mathrm{R}$ & 13 & $\mathrm{~S}$ & 16 & $\mathrm{~S}$ & 17 & $\leq 13$ & $14-15$ & $\geq 16$ \\
\hline 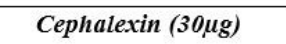 & $\mathrm{CL}$ & $\mathrm{R}$ & 14 & $\mathrm{R}$ & 13 & $\mathrm{R}$ & 12 & $\leq 14$ & $15-17$ & $\geq 18$ \\
\hline 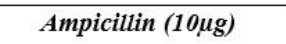 & AMP & $\mathrm{R}$ & 13 & $\mathrm{R}$ & 0 & $\mathrm{R}$ & 0 & $\leq 13$ & $14-16$ & $\geq 17$ \\
\hline Gentamicin $(10 \mu g)$ & $\mathrm{CN}$ & $\mathrm{R}$ & 12 & $\mathrm{~S}$ & 19 & $\mathrm{~S}$ & 20 & $\leq 12$ & 13-14 & $\geq 15$ \\
\hline Tetracycline $(30 \mu \mathrm{g})$ & $\mathrm{TC}$ & $\mathrm{S}$ & 15 & $\mathrm{R}$ & 10 & $\mathrm{R}$ & 11 & $\leq 11$ & $12-14$ & $\geq 15$ \\
\hline 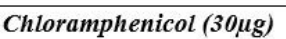 & $\mathrm{C}$ & $\mathrm{R}$ & 11 & $\mathrm{~S}$ & 21 & $\mathrm{~S}$ & 21 & $\leq 12$ & $13-17$ & $\geq 18$ \\
\hline
\end{tabular}

${ }^{1} \mathrm{R}=$ Resistant $;{ }^{2} \mathrm{I}=$ Intermediate $;{ }^{3} \mathrm{~S}=$ Susceptible

A. baumannii $\mathrm{W} 2$ was an MDRAB strain showing $M E M^{\mathrm{R}} C \mathrm{~L}^{\mathrm{R}} A M \mathrm{P}^{\mathrm{R}} \mathrm{CN}^{\mathrm{R}} \mathrm{TC}^{\mathrm{S}} \mathrm{C}^{\mathrm{R}}$ antibiotype. On the other hand, A. baumannii $\mathrm{C} 2$ and $A$. baumannii $\mathrm{C} 3$ was found to exhibit almost same antibiogram with the resistance to cephalexin, ampicillin, and tetracycline (Figure 1b, Figure 1c, and Table 2). The both isolates were also designated as MDRAB of MEM ${ }^{\mathrm{S}} C \mathrm{~L}^{\mathrm{R}} \mathrm{AMP}{ }^{\mathrm{R}} \mathrm{CN}^{\mathrm{S}} \mathrm{TC}^{\mathrm{R}} \mathrm{C}^{\mathrm{S}}$ antibiotype.

The resistance to $\beta$-lactams and cephalosporins showed by $A$. baumannii has been well documented [8, $15,32-34]$. Yang [8] attributed the resistance to $\beta$-lactams as a result including the production of $\beta$-lactamases, changes in penicillin-binding proteins that prevent activities of $\beta$-lactam drugs, alterations of porin proteins that result in decreased permeability to antibiotics, and the activity of efflux pumps that decreases the concentration of antibiotics within the bacteria. Previous studies have shown that a wide range of extended spectrum betalactamases could be found in A. baumannii clinical isolates [9]. Resistance to aminoglycosides was cited in several studies $[15,32,35,36]$.Similarly, tetracycline resistant $A$. baumannii is also not a new finding in clinical research $[15,37,38]$.

The overall findings of susceptibility assay are significant regarding prevention and control perspective, and onerous. The isolates' resistance to different antibiotics of multiple groups is an appalling finding of this investigation as totally working antibiotic options available to treat nosocomial infections with MDRAB are censoriously limited [15]. Moreover, MDRAB nosocomial infections are associated with a significant mortality percentage [7].

The presence of MDRAB among patients in our sampling healthcare set up further bolster the urgency of revision of hospital management system and implementation of necessary practices to limit the presence, cross-contamination and possible outbreaks of severe A. baumanii infections.

\section{Conclusion}

These findings alarm the onset of more devastating antimicrobial resistant catastrophe. The study also urges adequate hygienic practices in health care practices to curb prevalence and subsequent antimicrobial resistance of the formidable emerging pathogen.

\section{Declaration of conflict}

Kartik Dhar and Tareque Mahmud contributed equally.

\section{Acknowledgement}

The authors thank The University of Chittagong, Chittagong 4331, Bangladesh for funding. 


\section{References}

[1] Dijkshoorn, L., A. Nemec, and H. Seifert, An increasing threat in hospitals: multidrug-resistant Acinetobacter baumannii. Nature Reviews Microbiology, 2007. 5(12): p. 939-951.

[2] Roca, I., et al., The Acinetobacter baumannii oxymoron: commensal hospital dweller turned pan-drug-resistant menace. Analyzing possible intersections in the resistome among human, animal and environment matrices, 2015: p. 40.

[3] Lockhart, S.R., et al., Antimicrobial resistance among Gram-negative bacilli causing infections in intensive care unit patients in the United States between 1993 and 2004. Journal of clinical microbiology, 2007. 45(10): p. 3352-3359.

[4] Nordmann, P., Acinetobacter baumannii, le pathogène nosocomial par excellence.Pathologiebiologie, 2004. 52(6): p. 301-303.

[5] Morfin-Otero, R. and M.J. Dowzicky, Changes in MIC within a global collection of Acinetobacter baumannii collected as part of the Tigecycline Evaluation and Surveillance Trial, 2004 to 2009. Clinical therapeutics, 2012. 34(1): p. 101-112.

[6] Kempf, M. and J.-M. Rolain, Emergence of resistance to carbapenems in Acinetobacter baumannii in Europe: clinical impact and therapeutic options. International journal of antimicrobial agents, 2012. 39(2): p. 105-114.

[7] [Wilson, S.J., et al., Direct costs of multidrug-resistant Acinetobacter baumannii in the burn unit of a public teaching hospital. American journal of infection control, 2004. 32(6): p. 342-344.

[8] Yang, S.-C., et al., Prevalence of antibiotics resistance and OXA carbapenemases genes in multidrug-resistant Acinetobacter baumannii isolates in central Taiwan. European journal of clinical microbiology \& infectious diseases, 2010. 29(5): p. 601-604.

[9] Gordon, N.C. and D.W. Wareham, Multidrug-resistant Acinetobacter baumannii: mechanisms of virulence and resistance. International journal of antimicrobial agents, 2010. 35(3): p. 219-226.

[10] Esterly, J.S., et al., Genetic mechanisms of antimicrobial resistance of Acinetobacter baumannii. Annals of Pharmacotherapy, 2011. 45(2): p. 218-228.

[11] Neonakis, I.K., D.A. Spandidos, and E. Petinaki, Confronting multidrug-resistant Acinetobacter baumannii: a review. International journal of antimicrobial agents, 2011. 37(2): p. 102-109.

[12] Poirel, L., R.A. Bonnin, and P. Nordmann, Genetic basis of antibiotic resistance in pathogenic Acinetobacter species. IUBMB life, 2011. 63(12): p. 1061-1067.

[13] Zavascki, A.P., et al., Multidrug-resistant Pseudomonas aeruginosa and Acinetobacter baumannii: resistance mechanisms and implications for therapy. Expert review of anti-infective therapy, 2010. 8(1): p. 71-93.

[14] García-Garmendia, J.-L., et al., Risk factors for Acinetobacter baumannii nosocomial bacteremia in critically ill patients: a cohort study. Clinical infectious diseases, 2001. 33(7): p. 939-946.

[15] Perez, F., et al., Global challenge of multidrug-resistant Acinetobacter baumannii. Antimicrob Agents Chemother, 2007. 51(10): p. 3471-84.

[16] Qi, C., et al., Characterization of genetic diversity of carbapenem-resistant Acinetobacter baumannii clinical strains collected from 2004 to 2007. Journal of clinical microbiology, 2008. 46(3): p. 1106-1109.

[17] Jean, S.-S., et al., Nationwide surveillance of antimicrobial resistance among non-fermentative Gram-negative bacteria in Intensive Care Units in Taiwan: SMART programme data 2005. International journal of antimicrobial agents, 2009. 33(3): p. 266-271.

[18] Islam, M., et al., Emergence of multidrug-resistant NDM-1-producing Gram-negative bacteria in Bangladesh. European journal of clinical microbiology \& infectious diseases, 2012. 31(10): p. 2593-2600.

[19] Islam, T.A.B., S. Shamsuzzaman, and A. Farzana, Prevalence and antibiogram of ESBL producing gram negative bacilli isolated from urine in Dhaka Medical College Hospital, Bangladesh. Bangladesh Journal of Medical Microbiology, 2017. 9(1): p. 17-21.

[20] Islam, T.A.B., et al., Prevalence and Antibiogram of Microbial Agents Causing Nosocomial Urinary Tract Infection in Surgical Ward of Dhaka Medical College Hospital. Journal of Enam Medical College, 2016. 6(2): p. 75-79.

[21] Khatun, N., et al., Molecular characterization and resistance profile of nosocomial Acinetobacter baumannii in intensive care unit of tertiary care hospital in Bangladesh. Bangladesh Medical Research Council Bulletin, 2016. 41(2): p. 101-107.

[22] Mannan, M.A., et al., Microbiological Profile of Severe Lower Respiratory Tract Infection in Intensive Care Unit of a Tertiary Care Center of Dhaka, Bangladesh. Bangladesh Critical Care Journal, 2015. 2(2): p. 53-56.

[23] Guder, W., et al., Samples: from the patient to the laboratory. The impact of preanalytical variables on the quality of laboratory results. Darmstadt: GIT Verlag, 1996.

[24] Miller, J.M. and H.T. Holmes, Specimen collection, transport, and storage. 1999.

[25] Bayer, A., et al., Antibiotic susceptibility testing by a standardized single disc method. Am J clinpathol, 1966. 45(4): p. 493-496.

[26] Cockerill, F.R., Performance standards for antimicrobial susceptibility testing: twenty-first informational supplement. 2011: Clinical and Laboratory Standards Institute (CLSI).

[27] Villegas, M.V. and A.I. Hartstein, Acinetobacter Outbreaks, 1977-2000. Infection Control \& Hospital Epidemiology, 2003. 24(04): p. 284-295.

[28] Antunes, L.C., P. Visca, and K.J. Towner, Acinetobacter baumannii: evolution of a global pathogen. Pathogens and disease, 2014. 71(3): p. 292-301.

[29] Maza, L.M.d.1., et al., Color atlas of medical bacteriology. 2013, Washington: ASM Press. xi +353 pp.

[30] Buchanan, R.E., Bergey's manual of determinative bacteriology. 1974.

[31] Xiao-min, X., et al., Antibiotic resistance determinants of a group of multidrug-resistant Acinetobacter baumannii in China. The Journal of antibiotics, 2014. 67(6): p. 439-444.

[32] Lin, M.F., et al., Molecular epidemiology and antimicrobial resistance determinants of multidrug-resistant Acinetobacter baumannii in five proximal hospitals in Taiwan. Jpn J Infect Dis, 2011. 64(3): p. 222-7.

[33] Bakour, S., et al., Antibiotic resistance determinants of multidrug-resistant Acinetobacter baumannii clinical isolates in Algeria. Diagnostic microbiology and infectious disease, 2013. 76(4): p. 529-531.

[34] Biglari, S., et al., Antimicrobial Resistance Mechanisms and Genetic Diversity of Multidrug-Resistant Acinetobacter baumannii Isolated from a Teaching Hospital in Malaysia. Microbial Drug Resistance, 2016.

[35] Magnet, S., P. Courvalin, and T. Lambert, Resistance-nodulation-cell division-type efflux pump involved in aminoglycoside resistance in Acinetobacter baumannii strain BM4454. Antimicrobial agents and chemotherapy, 2001. 45(12): p. 3375-3380.

[36] Nemec, A., et al., Diversity of aminoglycoside-resistance genes and their association with class 1 integrons among strains of panEuropean Acinetobacter baumannii clones. Journal of medical microbiology, 2004. 53(12): p. 1233-1240.

[37] Huys, G., et al., Distribution of tetracycline resistance genes in genotypically related and unrelated multiresistant Acinetobacter baumannii strains from different European hospitals. Research in microbiology, 2005. 156(3): p. 348-355.

[38] Guardabassi, L., et al., Distribution and in-vitro transfer of tetracycline resistance determinants in clinical and aquatic Acinetobacter strains. Journal of medical microbiology, 2000. 49(10): p. 929-936. 